\title{
Determinação dos Salários num Modelo de Shirking-Matching com Informação Assimétrica
}

\author{
Rodolfo Ferreira Ribeiro da Costa ${ }^{1}$ \\ Isaac Ricarte Evangelista ${ }^{2}$ \\ Márcio Veras Corrêa ${ }^{3}$
}

\begin{abstract}
Resumo: O objetivo deste trabalho é apresentar a condição ótima para determinação do salário num modelo de Shirking-Matching onde o problema de moral hazard é presente. O cenário vigente é baseado num modelo de busca, onde as firmas e trabalhadores estabelecem o nível de salários através de um processo de barganha. As firmas são homogêneas e possuem livre entrada no mercado. Existem dois tipos de trabalhadores que vivem infinitamente: os educados/habilidosos e os não-educados/não-habilidosos. No equilíbrio, observa-se uma condição para equação de salário que deve proporcionar ao trabalhador um nível de renda superior o que seria obtido pelo mesmo quando ele se encontra na situação de desemprego. Ainda, a regra de determinação da função market tightness é dada pela seguinte condição: a contratação de um novo trabalhador deve gerar um fluxo de produto tal que o ganho proveniente de um posto de trabalho ocupado seja igual ao custo unitário de recrutamento descontado. Observou-se uma relação negativa entre o poder de barganha do trabalhador e a taxa de desemprego.
\end{abstract}

Palavras Chaves: Shirking, Matching, Risco Moral, Salários.

Classificação JEL: D82; J31; J64

1 Graduado em Economia pela UERN, Mestre em Economia pela UFPB, Doutor em Economia pelo CAEN/ UFC e Pós Doutorando em Economia pela UFRN. E-mail: rodolfofrc@yahoo.com.br

2 Graduado em Matemática pela UFC, Mestre em Economia pelo CAEN/UFC e Doutorando em Economia pelo CAEN/UFC. E-mail: isaac.evangelista@gmail.com

3 Graduado em Economia pela Unifor, Mestre em Economia pelo CAEN/UFC, Doutor em Economia pela Universidade Técnica de Lisboa - UTL e Pós Doutor pela University of Cambridge. E-mail: marciovcorrea@ 


\title{
Wage determination in a shirking -matching model with asymmetric information
}

\begin{abstract}
The purpose of this paper is to present the optimal condition for the determination of wages in Shirking-matching model where the problem of moral hazard is present. The current scenario is based on a Search Model, where firms and workers set the wage level through a process of bargaining. Firms are homogeneous and have free entry. There are two types of workers who live infinitely: the educated/skilled and no-educated/no-skilled. At equilibrium, there is an equation of condition for wages that must give the employee an income level above which would be obtained by even when he is in the unemployed. Still, the rule of determining the market tightness function is given by the following condition: hiring a new employee must produce a product stream such that the gain from a busy work station is equal to the discounted unit cost of recruitment. There was a negative relationship between the bargaining power of workers and the unemployment rate.
\end{abstract}

Key Words: Shirking, Matching, Moral hazard, Wages.

JEL Classification: D82; J31; J64

\section{Introdução}

Num ambiente de mercado observa-se um fluxo de contratações e demissões proporcionado pela interação econômica entre empregadores e empregados. Muito deste movimento diz respeito aos problemas de informação implícitos a relação. Questões referentes ao potencial do trabalhador, bem como a sua reputação, não são observadas pela firma contratante, de modo que o resultado gerado pelo matching pode ser ineficiente.

O ponto de partida ao se tratar deste assunto remete ao contrato que determina a jornada de trabalho e, principalmente, o salário praticado durante o período no qual a relação empregador-trabalhador for estabelecida. O objetivo deste trabalho é apresentar a condição ótima para determinação do salário num modelo de Shirking-Matching onde o problema de moral hazard é presente. O cenário vigente é baseado num mercado competitivo Walrasiano, onde as firmas e trabalhadores estabelecem o nível de salários através de um processo de barganha.

Este paper baseia-se nos trabalhos de Rocheteau (2002) e Charlot e Decreuse (2010). O primeiro utiliza um modelo de Shirking-Matching envolvendo problemas de risco moral para determinar a condição ótima que rege o montante da jornada de trabalho e os salários para um grupo de países europeus. Suas 
contribuições, baseadas num ambiente constituído por contínuo de agentes homogêneos (firmas e trabalhadores), procuram destacar que em países com alta taxa de desemprego, uma redução na jornada de trabalho promove uma variação negativa sobre a referida taxa. Ainda, os salários observados numa situação de livre negociação são superiores àqueles observados sobre considerações da teoria do salário eficiente. O segundo utiliza um modelo de Search-Matching com seleção adversa num cenário onde existe um continuo de firmas homogêneas e um conjunto de trabalhadores heterogêneos divididos em dois grupos: educados/habilidosos e não educados/não-habilidosos. De acordo com estes últimos autores, o fluxo de produto que é igual ao salário pago ao ocupante de um posto de trabalho depende do nível de escolaridade do empregado. Aquele setor que possui uma força de trabalho mais habilidosa, consequentemente, é mais produtivo e, assim, é capaz de oferecer um maior nível de salários. Por fim, trabalhadores não educados subvalorizam o investimento em educação, enquanto os trabalhadores educados sobrevalorizam tal tipo de investimento.

Neste trabalho tratar-se-á a relação de barganha salarial entre firmas e trabalhadores com níveis educacionais diferenciados e sobre um contexto de moral hazard. Assim, utilizar-se-á o modelo de Rocheteau (2002) levando em consideração a proposta de Charlot e Decreuse (2010), que enquadravam os trabalhadores de acordo com seu nível de habilidade.

Portanto, a contribuição deste artigo para literatura sobre mercado de trabalhado deve-se a análise da determinação do salário num ambiente com problemas de informação (Moral Hazard) e indivíduos com níveis de educação heterogêneos. Por fim, admite-se que os contratos são estacionários.

Além desta seção introdutória, o trabalho possui mais três seções. A próxima seção apresenta o modelo que será utilizada para tratar a relação entre firmas e trabalhadores, no que tange a determinação do salário, sobre informação assimétrica. A segunda proporciona de forma analítica a remuneração paga por posto de trabalho ocupado de acordo com o tipo especifico do trabalhador. Por fim, as considerações finais.

\section{O Modelo}

\subsection{Agentes}

A economia vigente é composta por firmas homogêneas que possuem livre entrada no mercado e dois tipos de trabalhadores que vivem infinitamente: os educados/habilidosos $(h)$ e os não-educados/não-habilidosos $(l)$. O 
tempo é denotado por $t$, os agentes descontam o valor de suas dotações a uma taxa $r \in \mathbb{R}^{+}$.

A utilidade do trabalhador $i$ é representada pela diferença entre a renda total $(x)$ e a desutilidade o trabalho $e(l)$ :

$$
U(x, l)_{i}=x_{i}-e(l)_{i}
$$

onde, $i$ refere-se ao $i$-ésimo tipo do trabalhador $(i=h, l)$. $l$ como subscrito refere-se ao tipo do trabalhador, enquanto que como variável, exprime o período de tempo destinado ao trabalho. Neste último caso $l \in[0,1]$. A desutilidade do trabalho apresenta as propriedades usuais: é estritamente convexa, crescente $e(0)=0$. De $(1)$, pode-se representar a utilidade do trabalhador $i$ descontada pela taxa $r$ como segue:

$$
\int e^{-r t} U[x(t), l(t)] d t
$$

O fluxo de produção, $y(l)$, apresenta retornos constantes e é estritamente côncava nas horas de trabalho. Segundo Hart (1987) quando o trabalhador é exposto a uma grande jornada de trabalho observa-se uma queda de rendimento promovida pelo efeito fadiga. Ainda, observam-se as seguintes propriedades para função de produção:

$$
y(0)=0, y^{\prime}(0)=\infty e y^{\prime}(\infty)=0, y^{\prime}(1)<e^{\prime}(1) \text { e } y^{\prime}(0)>e^{\prime}(0)
$$

A primeira igualdade é bastante óbvia. Não se pode produzir sem utilizar alguma unidade do insumo trabalho. As duas seguintes correspondem às condições de Inada. Por fim, as desigualdades implicam que $l \in(0,1)$ e que a desutilidade do trabalho é superior ao fluxo de produção quando o individuo enfrenta uma carga de trabalho igual a 24 horas.

A relação entre firmas e trabalhadores é dada pela função matching, $m(v, u)$ a qual exibe retornos constante de escala. $v$ refere-se ao número de vagas e $u$ diz respeito ao número de desempregados, ambos endógenos. Ainda, verifica-se que $m(0,0)=0$ e $m\left({ }^{\prime}, \infty\right)=m\left(\infty,{ }^{\prime}\right)=\infty$. A função market tightness, que descreve a razão entre $v$ e $u$, é dada por $\theta$. Desta definição, é possível determinar a taxa na qual as vagas são preenchidas e a taxa de saída da situação de desemprego, respectivamente:

$$
q(\theta)=m(v, u) / v \text { e } \theta q(\theta)=m(v, u) / u
$$


onde, $q^{\prime}()<0,. q(0)=\infty e q(\infty)=0$.

A elasticidade de $q($. ) e de $\theta q(\theta)$ em relação à $\theta$ é dada, respectivamente, por:

$$
\eta=\left(\frac{-q^{\prime}(\theta)}{q(\theta)}\right) \theta \in[0,1] e 1-\eta=\left(\frac{q(\theta)+\theta q^{\prime}(\theta)}{q(\theta)}\right)
$$

A destruição de um posto de trabalho é dada por um processo de Poisson com taxa de chega $\delta \in \mathbb{R}^{+}$. Este processo gera um fluxo de desemprego igual a $\delta(1-u)$. No estado estacionário observa-se que este fluxo deve ser igual ao fluxo de saída da situação de desemprego. Logo,

$$
\begin{gathered}
u \theta q(\theta)=\delta(1-u) \\
u=\left(\frac{\delta}{\delta+\theta q(\theta)}\right)
\end{gathered}
$$

e, portanto, a taxa de desemprego é decrescente em $\theta$.

\subsection{Problema de Moral Hazard}

Como a economia é composta por trabalhadores de dois tipos, $h$ e $l$, e a firma não observa o tipo de trabalhador que esta contratando, faz-se necessária uma proxy sobre as habilidades do trabalhador que ajude a identificar o seu tipo de comportamento. Por simplicidade, admita que as firmas observam o nível de escolaridade do trabalhador, sendo que aqueles trabalhadores com o nível de escolaridade mais alto, na média, são aqueles considerados educados e, portanto, sejam os mais produtivos.

O problema de moral hazard surge devido ao hiato de produto que pode ser observado dentro de um processo produtivo: o produto gerado por um trabalhador habilidoso, $y_{h}$, pode ser inferior a média dos trabalhadores educados, $y_{h}$, e, de forma semelhante, o produto criado pelos trabalhadores não-habilidosos, $y_{l}$, pode estar aquém da média produzida pelo seu tipo, $y_{l}$. Assim, a taxa de Shirking pode ser expressa como segue: 


$$
s=\left(\frac{\bar{y}\left(l_{i}\right)-y\left(l_{i}\right)}{\bar{y}\left(l_{i}\right)}\right)
$$

Enquanto o trabalhador está melhor fazendo $\mathrm{s}=1$, pois ele exerce um esforço menor e ganha o mesmo salário que os demais trabalhadores do seu tipo, a firma está melhor quando $\mathrm{s}=0$, pois o trabalhador do tipo $i$ exerce um esforço equivalente a média do seu grupo.

Para evitar tal transtorno, Shapiro e Stiglitz (1984) propõem um mecanismo de inspeção a ser realizado de acordo com um processo de Poisson com taxa de chegada dada por $\lambda \in \mathbb{R}^{+}$. Caso, $s>0$ o trabalhador do tipo $i$ será demitido. Portanto, além da probabilidade de demissão via destruição do posto de trabalho, $\delta$, existe a probabilidade de demissão via inspeção, $\lambda$.

\subsection{Função Valor do Trabalhador e da Firma}

A função valor do trabalhador $i$ satisfaz a seguinte equação de precificação de ativos:

$$
r v_{E i}\left(w_{i}\right)=\max _{s \in[0,1]}\left\{w_{i}-e\left((1-s) l_{i}\right)+\left(\lambda 1_{(0,1]}(s)+\delta\right)\left(v_{u i}-v_{E i}\left(w_{i}\right)\right)\right\}
$$

onde, $r v_{E i}$ é a utilidade esperada de um trabalhador empregado do tipo $i$, $v_{u i}$ é a utilidade esperada de um trabalhador desempregado do tipo $i, w_{i}$ é o salário real do trabalhador do tipo $i, 1_{(0,1]}(s)$ é uma função indicador que é igual a unidade quando o argumento é positivo e igual a zero caso contrário. A função valor do trabalhador do tipo $i$ destaca que ele recebe uma renda $w_{i} \mathrm{e}$, em contrapartida, incorre numa desutilidade dada por $e\left((1-s) y_{i}\right)$. Ainda, observa-se que ele possui uma probabilidade de se encontrar desempregado, caso o trabalho seja destruído, igual a $\delta \in \mathbb{R}^{+}$e, caso seja demitido, igual a $\delta \in \mathbb{R}^{+}$se $s>0$ e 0 caso contrário.

A estratégia ótima do trabalhador do tipo $i$ é escolher $s \rightarrow 0$ se e somente se:

$$
v_{E i}\left(w_{i}\right)-v_{u i} \geq \frac{e\left(l_{i}\right)}{\lambda}
$$


A expressão acima representa a condição No-Shirking (NSC). Ela retrata que o ganho de um trabalhador estar empregado deve ser superior a desutilidade do trabalho ponderada pela probabilidade de demissão.

De forma similar, a função valor do trabalhador do tipo $i$ que se encontra desempregado é dada como segue:

$$
r v_{u i}\left(w_{i}\right)=b+\theta q(\theta)\left(v_{E i}\left(w_{i}\right)-v_{u i}\right)
$$

onde $b \in \mathbb{R}^{+}$, é valor do seguro desemprego independente do tipo do trabalhador, o segundo termo representa o ganho de capital esperado.

A função valor da firma, denotada por $v_{j}\left(w_{i}\right)$, satisfaz a seguinte equação de precificação de ativos:

$$
r v_{j}\left(w_{i}\right)=\sum y\left(l_{i}\right)-\sum w_{i}+\delta \sum\left\{v_{v i}-v_{j}\left(w_{i}\right)\right\}
$$

O valor de uma vaga de trabalho, $v_{v i}$, é dada pela seguinte equação de Bellman:

$$
r v_{V i}=-\gamma+q(\theta)\left\{v_{j}\left(w_{i}\right)-v_{V i}\right\}
$$

onde, $\gamma$ é o custo de procura por um trabalhador, o qual é independente do tipo do trabalhador.

Tomando $v_{v i}=0$, temos que:

$$
v_{j}\left(w_{i}\right)=\frac{\gamma}{q(\theta)}
$$

Logo, em equilíbrio, o valor de uma vaga ocupada deve ser igual ao custo médio de recrutamento. Substituindo (9) em (7), obtém-se $\theta$ como uma função decrescente da renda real do trabalhador:

$$
y\left(l_{i}\right)-w=(r+\delta) \frac{\gamma}{q(\theta)}
$$




\subsection{Barganha de Nash}

O ambiente estudado neste trabalho baseia-se numa economia onde firmas e trabalhadores determinam o nível salarial de acordo com um processo de barganha. Uma vez determinado o salário do trabalhador do tipo $i, w_{i}$, chega-se a um contrato do tipo estacionário, ou seja, ele não muda em função do tempo.

De acordo com Rocheteau (2002), Pissarides (2000), Marimon e Zilliboti (2000) o processo de barganha deve atender a condição de No-Shirking, de modo que o mínimo salário deve ser tal que impeça o trabalhador de ser Shirking. O resultado da negociação não pode violar a NSC, caso contrário o excesso da empresa seria negativo. $O$ resultado do processo de barganha combina a solução de Nash assimétrica com pontos que iguala os valores respectivos do empregador e trabalhador num Search contínuo.

Com o poder de barganha do trabalhador do tipo $h$ e $l$ dados, respectivamente, por $\alpha, \beta \in(0,1)$, com $\alpha+\beta<1$, pode-se apresentar o problema de barganha como segue:

$$
\max _{w_{i} \in\left[0, y_{i}\right]}\left[v_{E h}\left(w_{h}\right)-v_{u h}\right]^{\alpha}\left[v_{E l}\left(w_{l}\right)-v_{u l}\right]^{\beta}\left[v_{j}\left(w_{i}\right)\right]^{1-\alpha-\beta}
$$

Sujeito a $v_{E i}\left(w_{i}\right)-v_{u i} \geq \frac{e\left(l_{i}\right)}{\lambda}, \operatorname{com} i=h, l$.

Esta restrição pode ser reescrita, utilizando as equações (4) e (6), como:

$$
w_{i} \geq(r+\delta) \frac{e\left(l_{i}\right)}{\lambda}+e\left(l_{i}\right)+r v_{u i}, \operatorname{com} i=h, l
$$

Admite-se que a restrição é ativa.

Uma condição necessária e suficiente para formação de um matching sobre transferência de utilidade é que:

$$
v_{E i}\left(w_{i}\right)-v_{u i}+v_{j}\left(w_{i}\right)=\frac{y\left(l_{i}\right)-e\left(l_{i}\right)-r v_{u i}}{r+\delta} \geq \frac{e\left(l_{i}\right)}{\lambda}
$$


A solução analítca do problema de barganha pode ser obtida utilizando as equações (4), (7) e multiplicando cada termo do produto de Nash por $(r+\delta)$. Assim, as condições de primeira ordem são dadas por:

$$
\begin{gathered}
\alpha\left(\frac{y\left(l_{h}\right)-w_{h}}{w_{h}-e\left(l_{h}\right)-r v_{u h}}\right)^{1-\alpha}-(1-\alpha-\beta)\left(\frac{w_{h}-e\left(l_{h}\right)-r v_{u h}}{y\left(l_{h}\right)-w_{h}}\right)^{1-\alpha-\beta}+\xi=0 \\
\beta\left(\frac{y\left(l_{l}\right)-w_{l}}{w_{l}-e\left(l_{l}\right)-r v_{u l}}\right)^{1-\beta}-(1-\alpha-\beta)\left(\frac{w_{l}-e\left(l_{l}\right)-r v_{u l}}{y\left(l_{l}\right)-w_{l}}\right)^{1-\alpha-\beta}+\xi=0 \\
\xi\left(w_{i}-(r+\delta) \frac{e\left(l_{i}\right)}{\lambda}+e\left(l_{i}\right) r v_{u i}\right)=0
\end{gathered}
$$

onde, $\xi$ é o multiplicador de lagrange associado com a condição de No-Shirking.

\section{Determinação do Nível de Salários}

Posto todo problema enfrentado pelas firmas e trabalhadores na divisão dos ganhos do matching através de um sistema de barganha de Nash, passa-se a determinação do nível de salário e, assim, do contrato de trabalho numa situação de informação assimétrica com moral hazard.

Sendo a restrição do problema de maximização dos ganhos do matching ativa, ou seja, considerando a condição de No-Shirking como binding, verifica-se a partir da equação (14) e (15) a seguinte expressão para a renda do trabalhador empregado:

$$
\begin{gathered}
w_{h}=(r+\delta) \frac{e\left(l_{h}\right)}{\lambda}+e\left(l_{h}\right)+r v_{u h} \\
w_{l}=(r+\delta) \frac{e\left(l_{l}\right)}{\lambda}+e\left(l_{l}\right)+r v_{u l}
\end{gathered}
$$

Assim, das equações (16) e (17), pode-se verificar que o salário do trabalhador do tipo $i, w_{i}$, é dado pelo fluxo de renda do trabalhador $i$ (primeiro termo do lado direito das equações), gerado pelo problema de moral hazard, e por seu salário de reserva (dois últimos termos do lado direito das equações). Ainda, não se identifica uma relação entre o poder de barganha do trabalhador do tipo $i$, bem como, da sua produtividade, sobre salários. 
A renda do trabalhador desempregado do tipo $i$ pode ser obtida da equação (6). Logo, o valor descontado recebido por um trabalhador desempregado é dado como segue:

$$
\begin{aligned}
& r v_{u h}\left(w_{h}\right)=b+\theta q(\theta) \frac{e\left(l_{h}\right)}{\lambda} \\
& r v_{u l}\left(w_{l}\right)=b+\theta q(\theta) \frac{e\left(l_{l}\right)}{\lambda}
\end{aligned}
$$

Substituindo a equação (18) em (16),

$$
w_{h}=(r+\delta) \frac{e\left(l_{h}\right)}{\lambda}+e\left(l_{h}\right)+b+\theta q(\theta) \frac{e\left(l_{h}\right)}{\lambda}
$$

Da mesma forma, substituindo a equação (19) em (17), temos

$$
w_{l}=(r+\delta) \frac{e\left(l_{l}\right)}{\lambda}+e\left(l_{l}\right)+b+\theta q(\theta) \frac{e\left(l_{l}\right)}{\lambda}
$$

Portanto, o salário do trabalhador do tipo $i$ depende positivamente da sua desutilidade sobre o trabalho e da taxa de saída da situação de desemprego. A partir de (10) e (20 e 21) obtém-se a condição de equilíbrio que determina o valor da função market tightness, $\theta$ :

$$
\xi=w_{i}-\phi y\left(l_{i}\right)-(1-\phi)\left(e\left(l_{i}\right)+r v_{u i}\right)
$$

com $i=h, l$.

Portanto, o salário do trabalhador do tipo $i$ depende positivamente da sua desutilidade sobre o trabalho e da taxa de saída da situação de desemprego. O salário de equilíbrio oferecido ao trabalhador do tipo $i$ deve ser tal que o seu valor compense a sua desutilidade por trabalhar e ao valor recebido por não estar empregado.

Proposição 1 - Num equilíbrio de busca, assumindo a condição de $N o$ -Shirking, a quantidade de trabalho realizada pelo trabalhador do tipo $i$ é 
superior ao observado na ausência do Shirking.

Prova - De acordo com a condição de primeira ordem do problema de barganha, temos:

$$
\xi=w_{i}-\phi y\left(l_{i}\right)-(1-\phi)\left(e\left(l_{i}\right)+r v_{u i}\right)
$$

onde, $\xi=\left(y\left(l_{i}\right)-w_{i}\right)^{\phi}\left(w_{i}-e\left(l_{i}\right)-r v_{u i}\right)^{(1-\phi)} e \phi=\alpha+\beta$

Substituindo as equações (20) e (21) em (22),

$$
\xi=(r+\delta) \frac{e\left(l_{i}\right)}{\lambda}+e\left(l_{i}\right)+b+\theta q(\theta) \frac{e\left(l_{i}\right)}{\lambda}-\phi y\left(l_{i}\right)-(1-\phi)\left(e\left(l_{i}\right)+r v_{u i}\right)
$$

Por (21) e (22),

$$
\begin{gathered}
\xi=(r+\delta) \frac{e(l i)}{\lambda}+e(l i)+b+\theta q(\theta) \frac{e(l i)}{\lambda}-\phi y(l i)-e(l i)-b-\theta q(\theta) \frac{e(l i)}{\lambda}+\phi(e(l i)+r v u i) \\
\xi=(r+\delta) \frac{e\left(l_{i}\right)}{\lambda}-\phi y\left(l_{i}\right)+\phi\left(e\left(l_{i}\right)+r v_{u i}\right) \\
\frac{\xi}{(r+\delta)}=\frac{e\left(l_{i}\right)}{\lambda}-\frac{\phi y\left(l_{i}\right)+\phi\left(e\left(l_{i}\right)+r v_{u i}\right)}{(r+\delta)}
\end{gathered}
$$

De (12), temos que $\xi \leq 0$. Mas isto contradiz que a condição de No-Shirking é binding. Assim, a quantidade de produto gerado com o atendimento da condição de No-Shirking é superior ao montante produzido quando o Shirking é observado. Consequentemente, um produto maior é obtido por uma maior quantidade de trabalho empregada pelo trabalhador do tipo $i$.

Este trabalho coloca a firma com um agente capaz de influenciar o salário e mostra que não é simplesmente aumentar o nível educacional para que o salário do trabalhador aumente como observado por Charlot e Decreuse (2010). Verifica-se que com uma firma implantando um sistema de compatibilidade em incentivos e participação, ela exerce influência sobre o nível salarial do trabalhador do tipo $i$. 
Segundo Spence (1973) o retorno esperado sobre o investimento em educação para indivíduos com baixas qualidades é negativo, pois os mecanismos de determinação dos salários pelas firmas proporcionam um nível salarial que é inferior ao custo de um trabalhador com pouca qualificação se tornarem educados. Este resultado vai de encontro com os obtidos neste trabalho, os quais se contrapõem aos observados por Charlot e Decruese (2010).

A partir de (10) e (19) obtém-se a condição de equilíbrio que determina o valor da função market tightness, $\theta$ :

$$
(r+\delta) \frac{\gamma}{q(\theta)}=y\left(l_{i}\right)-(r+\delta) \frac{e\left(l_{i}\right)}{\lambda}-e\left(l_{i}\right)-b-\theta q(\theta) \frac{e\left(l_{i}\right)}{\lambda}
$$

$\operatorname{com} i=h, l$.

A partir de (21), identifica-se a razão entre o número de postos de trabalhados ofertados e o número de trabalhadores desempregados. No equilíbrio, o valor descontado do custo unitário de recrutamento deve ser igual à diferença entre o produto gerado pelo trabalhador do tipo $i$, que oferece $l_{i}$ horas de trabalho diário, e o custo de um posto de trabalho ocupado - formado pela soma entre a renda descontada para que o trabalhador se encontre melhor numa situação de empregado, o valor da sua desutilidade de trabalhar e o valor descontado recebido numa situação de desemprego.

De outra forma, a contratação de um novo trabalhador deve gerar um fluxo de produto tal que o ganho proveniente de um posto de trabalho ocupado seja igual ao custo unitário de recrutamento descontado. Este resultado segue a análise padrão realizada para determinação da quantidade de trabalho a ser contratada. Caso o resultado marginal da ocupação de um posto de trabalho seja positivo, a firma estaria melhor se aumentasse a quantidade de mão-de-obra. Caso contrário, se o resultado marginal for negativo, a firma encontrar-se-ia numa situação mais favorável optando por demitir alguns trabalhadores. Logo, tem- se o equilíbrio quando o resultado marginal é nulo.

De (23) e (24), temos:

$$
\begin{aligned}
& (r+\delta) \frac{\gamma}{q(\theta)}=y(l i)-\xi-\phi y(l i)+\phi(e(l i)+r v u i)-e(l i)-b-\theta q(\theta) \frac{e(l i)}{\lambda} \\
& (r+\delta) \frac{\gamma}{q(\theta)}=y(l i)-\xi-\phi y(l i)+\phi\left(e(l i)+b+\theta q(\theta) \frac{e(l i)}{\lambda}\right)-e(l i)-b-\theta q(\theta) \frac{e(l i)}{\lambda} \\
& (r+\delta) \frac{\gamma}{q(\theta)}=(1-\phi)\left(y\left(l_{i}\right)-e\left(l_{i}\right)-b-\theta q(\theta) \frac{e\left(l_{i}\right)}{\lambda}\right)-\xi
\end{aligned}
$$

Proposição 2 - Num equilíbrio de busca, assumindo a condição de No-Shirking, uma redução no poder de barganha do trabalhador leva a uma 
redução da taxa de desemprego.

Prova - A curva de oferta de postos de trabalho, dada por (25), que é ascendente na vizinhança do equilíbrio de busca, é afetada por uma modificação de $\phi$.

Portanto, o poder de barganha dos trabalhadores afeta positivamente o número de vagas de trabalho demandada pelas firmas. Este resultado é diferente do verificado por Rocheteau (2002), que identificou uma correlação nula entre o poder de barganha dos trabalhadores e o número de postos de trabalho criados pelas firmas.

O equilíbrio de busca é dado pelo par $\left(l_{i}, \theta\right)$ tal que (24) é satisfeita. A existência e unicidade do equilíbrio de busca são demonstradas a seguir:

Preposição 3 - Sobre a seguinte condição,

$$
y\left(l_{i}^{*}\right)-(r+\delta) \frac{e\left(l_{i}^{*}\right)}{\lambda}-e\left(l_{i}^{*}\right)-b-\theta q(\theta) \frac{e\left(l_{i}^{*}\right)}{\lambda}>0
$$

o equilíbrio de busca com $l_{i} \theta>0$ existe e é único.

Prova - Existência: Admita que $\theta=0$, ou seja, não existe recrutamento. Portanto, a condição de No-Shirking é binding e por (25), temos que $l_{i}^{*}=l_{i}$. De (26), o lucro por posto de trabalho ocupado (o produto menos o salário) é estritamente positivo. Assim, as firmas têm incentivos para entrar no mercado.

Unicidade: A intersecção entre a curva de oferta de postos trabalho (V) e a curva de trabalho ofertada pelos trabalhadores (L) estão no mesmo lado do lócus de separação (S). De acordo com (25), L está situado abaixo do lócus de separação quando $l_{i}^{*} \leq l_{i}$ e acima caso contrário. De acordo com (24), $L$ está situado abaixo do lócus de separação quando $\theta \leq \bar{\theta}$ e acima caso contrário.

Dois casos podem ser distinguidos:

A interseção de $V$ e $L$ está localizada abaixo do lócus de separação. $V$ só pode cruzar o lócus de separação para valores de $l_{i}$ menores que $l_{i}^{*}$. Suponha que $V$ e $L$ se cruzam para algum $l_{i}^{*} \leq l_{i}$. Para todo $l_{i}^{*} \leq l_{i}, V$ é negativamente inclinada e $L$ está situado acima do lócus de separação. Além disso, $S$ vai para o infinito e $V$ vai para 0 quando $l_{i} \rightarrow \infty$. Como conseqüência, $V$ intercepta o lócus de separação para algum $l_{i}^{*} \leq l_{i}$. Mas isto é impossível de acordo com a afirmação anterior. Assim, $V \cap L$ é dada para algum $l_{i}^{*} \leq l_{i}$.

(ii) A interseção de $V$ e $L$ está localizada acima do local de separação. 
$\hat{V}$ e $V$ cruzam o lócus de separação para alguns $l_{i}^{*} \leq l_{i}$. Para todo $l_{i}^{*} \leq l_{i}, V$ é negativamente inclinada e $L$ está situado acima do lócus de separação. Consequentemente, a intersecção de $V$ e $L$ também está localizada acima do local de separação.

A equação (26) afirma que uma condição necessária e suficiente para as empresas criem postos de trabalho é que os lucros esperados de um posto de trabalho ocupado, na ausência de qualquer congestionamento de outras empresas $(\theta=0)$, sejam positivos.

\section{Considerações Finais}

Analisando uma economia formada por trabalhadores heterogêneos e firmas idênticas, onde a formação dos salários é determinar de acordo com o processo de barganha entre empregadores e empregados, este trabalho se propôs determinar o nível de salários dos trabalhadores educados/habilidosos e não-educados/não-habilidoso baseado num modelo de Shirking-Matching num cenário com informação assimétrica com moral hazard.

No equilíbrio, observa-se uma condição para equação de salário, referente ao trabalhador do tipo $i$, onde o montante oferecido ao ocupante do posto de trabalho, atendendo a condição de No-Shirking, deve proporcionar ao mesmo um nível de renda superior o que seria obtido pelotrabalhador quando ele se encontra na situação de desemprego.

Como o trabalhador do tipo educado possui habilidades superiores ao trabalhador do tipo não-educado, um posto de trabalho ocupado por um educado proporciona um nível de produto médio superior ao observado quando o ocupante do cargo é não-educado. Consequentemente, o salário praticado entre os dois tipos de indivíduos deve ser diferente, sendo que o custo de um posto de trabalho ocupado por um trabalhador do tipo educado é superior ao observado quando a vaga é ocupada por um trabalhador do tipo não-educado.

Ainda, investir em educação não é condição suficiente para que a firma ofereça um nível salarial mais elevado como destacado por Charlot e Decreuse (2010). A realização de inspeções para verificar o comportamento produtivo do trabalhador é questão fundamental para determinação dos salários. Segundo Laffont e Martimort (2001), o mecanismo que é compatível em incentivos e atende a condição de participação é fator decisivo para determinação do valor oferecido pela firma a cada trabalhador. Contudo, aquele trabalhador de investir uma maior quantidade de recursos na sua formação tem maior probabilidade de adquirir uma maior recompensa. 
A função market tightness, baseada na condição de equilíbrio apresentada na equação (20), destaca que a razão entre o número de vagas criadas e a quantidade de desempregados aumenta quando o valor gerado por uma nova contratação supera o custo médio de procura descontado. Por outro lado, quando o custo médio de procura é superior aos ganhos advindos de uma nova contratação, tem-se uma variação na criação de postos de trabalho inferior a observada pela taxa de desemprego e, assim, ocorrerá uma redução no parâmetro $\theta$.

Ainda, destaca-se o efeito positivo do poder de barganha do trabalhador sobre a criação de novos postos de trabalho, proporcionando redução da taxa de desemprego. Resultado este não observado por Rocheteau (2002).

Por fim, recomenda-se, como uma extensão natural deste artigo, a caracterização dos trabalhadores através de um contínuo de tipos e/ou das firmas de forma heterogêneas.

\section{Referências}

Charlot, O.; Decreuse, B. (2010). Over-education for the rich, under-education for the poor: A search-theoretic microfoundation. Labour Economics. $\mathrm{n}^{0} .17$, p. 886-896, 2010.

Charlot, O.; Decreuse, B. (2005). Self-selection in education with matching frictions. Labour Economics. $\mathrm{n}^{0} .15$, p. 251-267, 2005.

Gibbons, R. (1992). Game theory for applied economists. Princeton University Press. 1992.

Hart, R.A. (1987). Working time and employment. Allen and Unwin, London. 1987.

Laffont, J. J.; Martimort, D. (2001). The theory of incentives: the principal-agent model. Princeton University Press. 2001.

Marimon, R.; Zilibotti, F. (2000). Employment and distributional effects of restricting working time. European Economic Review. nº. 44, p. 1291-1326, 2000.

Mas-Colell, A.; Whinston, M. D.; Green, J. L. (1995). Microeconomic theory. Oxford University Press. 1995.

Pissarides, C. A. (2000). Equilibrium unemployment theory. 2nd Edition. MIT Press, Cambridge. 2000.

Rocheteau, G. (2002). Working time regulation in a search economy with worker moral hazard. Journal of Public Economics. nº. 84, p. 387-425, 2002.

Shapiro, C.; Stiglitz, J. (1984). Equilibrium unemployment as a discipline device. American Economic Review. $\mathrm{n}^{\mathrm{o}}$. 74, p. 433-444, 1984.

Spence, A. M. (1973). Job market signaling. Quarterly Journal of Economics. ${ }^{\circ}$. 87, p. 355-374, 1973. 